\title{
Annäherung statt Mitgliedschaft? Die Schweiz und die EU nach den zweiten bilateralen Abkommen
}

\author{
René Schwok und Stephan Bloetzer*
}

\section{Bestätigung des dritten Wegs zwischen EU-Beitritt und Isolation}

Frankreich sagt Nein zum Europäischen Verfassungsvertrag. Die Schweiz stimmt einem zweiten bilateralen Annäherungsschritt an die Europäische Union zu. Sind die Eidgenossen europafreundlicher geworden und die Franzosen EU-feindlicher? Ist die Welt auf den Kopf gestellt? Keineswegs, stellt eine genauere Betrachtung klar. Es ist nicht das Thema dieses Beitrags, das französische Abstimmungsresultat zu untersuchen. Das Abstimmungsverhalten der Schweizer am 5. Juni hingegen darf nicht falsch ausgelegt werden. Ihre Unterstützung für eine Assoziierung der Schweiz an das Schengen- und Dublin-System ist kein Blankoscheck für einen EU-Beitritt. Er bestätigt den Willen der Mehrheit im Lande, sich der Union weiterhin anzunähern, ohne ihr beizutreten. Der Ausbau des dritten Wegs zwischen einer europäischen Randposition und der Vollmitgliedschaft ist das Ziel.

Das Referendum vom 5. Juni 2005 betraf die Assoziierung an das Schengen- und DublinSystem. Das Abkommen über die Assoziierung zum Schengen-Raum ist Teil eines neun bilaterale Verträge umfassenden Pakets, welches als ,zweite bilaterale Abkommen ' bezeichnet wird. Diese zweiten bilateralen Verträge nähern die Schweiz der Europäischen Union an und räumen einige Hindernisse auf dem Weg in Richtung eines EU-Beitritts beiseite. Doch es bleiben zahlreiche Schwierigkeiten bestehen. Zudem bestätigen diese Abkommen in den Augen der Schweizer, dass man sich selektiv in die Union integrieren kann, ohne Vollmitglied werden zu müssen. Ausserdem hat die Europäische Union zum ersten Mal in Beziehungen mit einem Drittstaat zu rechtlich verbrieften Ausnahmen bezüglich des Gemeinschaftsacquis Hand geboten. Die Schweiz müsste diese das Bankgeheimnis betreffenden Ausnahmen im Fall eines EU-Beitritts aber höchstwahrscheinlich wieder aufgeben.

Im ersten Teil dieses Beitrags versuchen wir zu erklären, warum die Schweiz seit der Gründung der Europäischen Gemeinschaft für Kohle und Stahl (EGKS) im Jahr 1951 der europäischen Einigung so misstrauisch gegenübersteht. Im zweiten Teil zeigen wir am Beispiel der zweiten bilateralen Abkommen wie die Schweiz mit selektiven Verträgen eine Ausgrenzung aus dem politischen System EU-Europas zu verhindern versucht. Anhand der Darstellung dieser Verträge wird ebenfalls sichtbar, dass paradoxerweise die bilaterale Vorgehensweise einerseits die Schweiz der Europäischen Union annähert, andererseits aber einen Schweizer EU-Vollbeitritt unwahrscheinlicher werden lässt.

\section{Die Gründe für den Schweizer Euroskeptizismus}

$\mathrm{Zu}$ unserer Vorgehensweise: Wir versuchen, die Schweizerische Situation zu verstehen, nicht sie zu begründen oder gar zu verteidigen. Wir hätten nichts gegen einen gut verhandelten EU-Beitritt der Schweiz einzuwenden. Wir sind auch der Meinung, dass viele Eidgenos-

Dr. René Schwok, Associate Professor, Abteilung für Politische Wissenschaft der Sozialwissenschaftlichen Fakultät sowie des Europainstituts der Universität Genf.

Stephan Bloetzer, lic. iur, M.E.S. des Europainstituts der Universität Genf, Doktorand an der Rechtswissenschaftlichen Fakultät der Universität Genf, Visiting Researcher am European University Institute, Florence 2003-2004. 
sen die Nachteile bezüglich einer EU-Mitgliedschaft übertreiben. Zudem haben wir bereits andernorts den wirtschaftlichen, politischen und die Schweizer Identität betreffenden Preis des Abseitsstehens des Landes von der europäischen Integration untersucht und dargestellt. ${ }^{1}$ Die Hauptfrage einer nicht-schweizerischen Leserschaft, warum die Schweiz der einzige Staat in Mittelwesteuropa bleibe, welcher der Europäischen Union nicht beitritt, obwohl er dies ohne weiteres tun könnte, ist jedoch aktueller und berechtigter denn je.

Wir untersuchen die zwiespältige Haltung der Schweiz gegenüber einem Beitritt zur Union im Hinblick auf vier Deutungsmuster: die Abwesenheit eines historischen Traumas, das Paradox der Neutralität, das politische System und die direkte Demokratie sowie die wirtschaftlichen Eigenarten des Landes. In der Wirklichkeit verzahnen sich diese vier Kategorien und bedingen sich gegenseitig. Sie sollen hier als Untersuchungs- und nicht als abschliessende Erklärungsmuster dienen.

\section{Die Abwesenheit eines historischen Traumas}

Im Gegensatz zu den anderen europäischen Ländern hat die Schweiz in der jüngeren Geschichte keine traumatischen Ereignisse erlebt. Sie musste keinen Weltkrieg, keine Diktatur und keine fremde Besatzung ertragen. Deshalb erachten die meisten Schweizer es nicht als notwendig, ihre staatliche Eigenständigkeit infrage zu stellen und mit einem EU-Beitritt auf einen Teil ihrer Souveränität zu verzichten. Die Erschütterungen durch die Weltkriege sind die Hauptfaktoren für die Abwendung der Europäer vom extremen Nationalismus und für die Einsicht in die Notwendigkeit der europäischen Einigung. Alle Studien zu den Ursprüngen der europäischen Integration zeigen, dass der Wunsch eines ,einigen Europa‘ eng mit dem Leiden während der Weltkriege verbunden ist. Die Erfahrung des Krieges hat die Gründerstaaten der Europäischen Gemeinschaften dazu veranlasst, ihre Beziehungen auf eine neue Grundlage zu stellen. Ihre Eliten und Völker haben folgende Lehren aus der Geschichte gezogen: Erstens ist Krieg furchtbar. Alles muss unternommen werden, um ihn unter Europäern unmöglich zu machen. Zweitens muss mit einer Dosis Supranationalismus die potenziell aggressive Nationalstaatlichkeit gebändigt werden. Drittens ist die Neutralität eine gefährliche Versuchung, welche keine Sicherheit gewährleistet. Viertens können die kleinen Staaten einen neuen französisch-deutschen Krieg nur verhindern, wenn sie vorschlagen, eine Sicherheitsgemeinschaft im Sinne von Karl Deutsch zu gründen, die Frankreich und Deutschland dauerhaft versöhnt und in eine supranationale Organisation einbindet. Fünftens: Der wirtschaftliche Protektionismus, wie er in den 1930er Jahren grassierte, muss ebenfalls verhindert werden, weil er den Nationalismus schürt.

Diese Einsichten wurden von den Schweizern nur teilweise mitvollzogen. Der Zweite Weltkrieg hat sie sogar in der Berechtigung ihrer nationalen Überzeugung und der Richtigkeit ihres politischen Systems bestärkt.

Der Zweite Weltkrieg hatte auch wirtschaftliche Konsequenzen. Nach dem Krieg war die Schweiz unsäglich reicher als ihre Nachbarn. Während vieler Jahre trennte ein Wohlstandsgraben die verschonte Schweiz vom kriegsversehrten Europa. Die wenigsten Schweizer waren bereit, ihr Wohlergehen mit den Ärmsten in Form der europäischen Integration zu teilen. Wie kein Ereignis zuvor hat der Zweite Weltkrieg die Einheit der Schweiz gestärkt. Im Gegensatz zu Belgien, wo während des Krieges starke Spannungen zwischen den Sprachgruppen ausgebrochen waren, haben sich die Schweizer Landesteile vereint, um dem „Dritten

1 René Schwok: Switzerland and the European Common Market, New York 1991. 
Reich" und dem faschistischen Italien gemeinsam zu widerstehen. ${ }^{2}$ Erwähnenswert ist zum Beispiel der immer noch in Kraft stehende Pakt des sozialen Friedens von 1938, welcher eingeführt wurde, um soziale Konflikte zu vermeiden. Auch der Eintritt der Sozialistischen Partei in die Bundesregierung von 1943 ist zu vermerken.

Die Erinnerung an die Mobilmachung während des Krieges hat sich natürlich mit der Zeit verflüchtigt. Trotzdem ist die Mehrheit der schweizerischen Bevölkerung noch nicht dazu zu bewegen, einen so positiven Geschichtsverlauf und den Erfolg des Schweizerischen politischen Modells grundlegend infrage zu stellen.

\section{Das Paradox der Neutralität}

Die Neutralität ist als zweiter Grund des schweizerischen Euroskeptizismus zu nennen. Für die Mehrheit der Schweizer ist sie ein großes Hindernis für einen EU-Beitritt. Sie sehen in ihr einen Hauptpfeiler der Aussenpolitik ihres Landes seit mehr als zweihundert Jahren. Die Neutralität wird nicht als ein überholtes Konzept eingestuft. In diesem Punkt tritt der Unterschied zwischen den Schweizern und den Belgiern und Niederländern, deren Neutralität sie nicht vor der deutschen Invasion bewahrt hatte, offen zu Tage. Letztere warfen die Neutralität nach dem Zweiten Weltkrieg konsequent über Bord.

Auch wenn die Europäische Union in ihren Rängen sechs neutrale oder nicht allianzgebundene Staaten zählt (Österreich, Finnland, Irland, Schweden, Malta und Zypern), sind viele Schweizer der Meinung, ein EU-Beitritt sei mit ihrer Neutralität nicht vereinbar. ${ }^{3}$ Dabei stellt sie kein objektives Hindernis für einen EU-Beitritt dar. In der Tat ist die Union kein Militärbündnis wie die NATO. Alle Entscheidungen betreffend die Verteidigung können mit einem Veto eines Mitgliedstaates belegt werden. Kein Mitgliedstaat kann zur Teilnahme an einem militärischen Einsatz gemäss der Petersberger Erklärung gezwungen werden. Und der Status der neutralen und nicht allianzgebundenen EU-Staaten ist nie angezweifelt worden. Auch die schweizerische Neutralität würde von der Union nicht infrage gestellt werden. Trotzdem könnte die Neutralitätsproblematik in naher Zukunft vermehrt in den Vordergrund treten, wenn die Union über eine rasche Eingreiftruppe verfügen wird, und sie zudem entscheidet, im Bedarfsfall auf NATO-Ressourcen zurückzugreifen, und ihren Einsatz damit quasi von einer Genehmigung der Vereinigten Staaten abhängig macht.

Schließlich ist die Verankerung der Neutralität im Volk auch gefühlsbedingt. ${ }^{4}$ Nur wenige Schweizer sind in der Lage, die Neutralität juristisch zu definieren, geschweige denn das Neutralitätsrecht von der Neutralitätspolitik zu unterscheiden. So ist die Neutralität eine Art Tabu, über das man nicht diskutiert. Die Eidgenossen werden jährlich mehrmals zu Abstimmungen an die Urne gerufen. Bezeichnenderweise haben noch nie hunderttausend Schweizer ihr Initiativrecht wahrgenommen, um die Abschaffung oder Anpassung der Neutralität an die neue außen- und sicherheitspolitische Lage zu verlangen. Und als die Schweizer sich im März 2002 endlich zum UNO-Beitritt durchrangen, war dies erst möglich geworden, nachdem die Regierung ihnen versprochen hatte, dass die Neutralität bewahrt würde.

2 Das hat manche Schweizer nicht davon abgehalten, die antikommunistischen und antisemitischen Ansichten des Nazismus zu teilen und mit dem Dritten Reich in einem Maß wirtschaftlich zusammen zu arbeiten, welches die Notwendigkeit des Überlebens der Schweiz weit überstieg.

3 Vgl. hierzu Laurent Goetschel: Die Zukunft der Neutralität im Rahmen der GASP: Widerspruch, Gleichgültigkeit oder Ergänzung, in: Thomas Cottier/Alwin R. Kopse (Hrsg.): Der Beitritt der Schweiz zur Europäischen Union: Brennpunkte und Auswirkungen, Zürich 1998, S. 637-662; Silvan Frik: Ist die schweizerische Sicherheitspolitik europafähig? Die Neutralität im Lichte der Entwicklungen der ESVP, Chur 2002, 312 S.

4 Alle Meinungsumfragen seit über vierzig Jahren zeigen konstant, dass eine überwältigende Mehrheit der Schweizer (zwischen 70 und 80 Prozent) an ihr festhalten will. 


\section{Das politische System der Schweiz, und die direkte Demokratie}

Auf den ersten Blick stellt das politische System der Schweiz kein Hindernis für einen EUBeitritt dar. Auch die Schweiz ist grundsätzlich eine parlamentarische Demokratie und die Kompetenzabtretungen des Bundes auf die Gemeinschaftsebene wären mit denjenigen der anderen Mitgliedstaaten vergleichbar. Das ,Demokratie-Defizit', wenn es denn besteht, wäre nicht grösser als für jeden anderen Mitgliedstaat. Auch die direkte Demokratie als solche ist keine unüberwindliche Hürde. Die Volksrechte blieben in allen vom Gemeinschaftsrecht nicht betroffenen Gebieten bestehen. Studien haben gezeigt, dass die überwiegende Mehrheit der kantonalen und bundesweiten Initiativ- und Referendumsabstimmungen auch im Falle einer Schweizer EU-Mitgliedschaft durchgeführt werden könnten. ${ }^{5}$ Weniger als zehn Prozent der einer Bundesabstimmung unterzogenen Vorlagen hätten inhaltlich angepasst werden müssen oder hätten bei einer Mitgliedschaft in der Union gar nicht dem Volk unterbreitet werden können. Über neunzig Prozent der Bundesvorlagen und beinahe hundert Prozent der Abstimmungen auf kantonaler und kommunaler Ebene könnten beibehalten werden.

Trotzdem wird die Schweiz an ihrer Annäherung an die Union durch die direkte Demokratie gehindert. Zusammengefasst bestehen diesbezüglich vier Hauptschwierigkeiten: Erstens ist die Schweiz das einzige Land der Welt mit so intensiver direktdemokratischer Beteiligung auf allen Regierungs- und Verwaltungsebenen. Man kann sowohl auf der Stufe der Gemeinde als auch des Kantons und des Bundes praktisch über jedes Thema abstimmen. Zudem ist der Föderalismus eng mit der direkten Demokratie verflochten. Das Land ist in sechsundzwanzig Kantone und Halbkantone aufgeteilt. Der Föderalismus verteilt die Macht auf diese verschiedenen Entscheidungsebenen. Die Kantonsparlamente und -regierungen achten sorgfältig auf ihre Rechte und treten nur sehr widerwillig Teile davon an ,Bern', geschweige denn ,Brüssel` ab. Der schweizerische Zentralstaat wird oft als bürokratisch und bürgerfern eingestuft, und die Europäische Union erst recht. Mit einer gewissen Berechtigung befürchten somit die Schweizer die Beschneidung ihrer sehr weitgehenden politischen Rechte. Zweitens benötigt ein EU-Beitritt und auch derjenige zum Europäischen Wirtschaftsraum (EWR) die Zustimmung nicht nur der Mehrheit der stimmenden Bürger, sondern auch einer Mehrheit der Kantone (ein so genanntes ,doppeltes Mehr'). Da die Europa-Gegner sich in den schwach bevölkerten, ländlich geprägten deutschschweizerischen Kantonen konzentrieren, ist anzunehmen, dass auf Bundesebene ungefähr eine 56-prozentige Mehrheit der Volksstimmen notwendig ist, um diese Abstimmungshürde zu nehmen. ${ }^{6}$ Drittens durchdringt die direkte Demokratie das gesamte auf Konkordanz und Konsens basierende politische System der Schweiz. ${ }^{7}$ Sogar die Zusammensetzung der Bundesregierung, Bundesrat ge-

$5 \quad$ Vgl. Insbesondere: Stephan Koerkemeyer: Direkte Demokratie und europäische Integration: Zu den Möglichkeiten und Grenzen unmittelbarer Volksbeteiligung an der staatlichen Willensbildung in der Europäischen Union, dargestellt am Beispiel der Schweiz, unter der Berücksichtigung der Rechtslage in den derzeitigen EUMitgliedstaaten, Bern/Zürich 1995; Andreas Kellerhals/Andreas Auer/Bertil Cottier/Nicole Mathé: Staatsrechtliche Auswirkungen einer Mitgliedschaft in der Europäischen Union - Vier Studien im Rahmen des Integrationsberichts 1999 (Schweiz - Europäische Union) des Bundesrates, Zürich 1999; des Weiteren: Astrid Epiney/Karine Siegwart (Hrsg.): Direkte Demokratie und Europäische Union, Editions universitaires, Freiburg/Üchtland, 1997; Pascal Mahon/Christophe Mueller: Adhésion de la Suisse à l'Union européenne et démocratie directe, in: Thomas Cottier/Alwin R. Kopse (Hrsg.): Der Beitritt der Schweiz zur Europäischen Union: Brennpunkte und Auswirkungen, Zürich 1998, S. 449-485.

6 Vgl. auch weiter unten unsere Ausführungen zur Abstimmung vom 5. Juni 2005 über die Assoziierung der Schweiz an das System von Schengen und Dublin.

7 Vgl. im Allgemeinen z.B. Wolf Linder: Swiss democracy: possible solutions to conflict in multicultural societies, London/New York 1994; und insbesondere in Bezug auf einen EU-Beitritt der Schweiz: Frédéric Esposito: La démocratie directe et l'intégration européenne: une barrière juridique ou identitaire?, in: René Schwok/ Nicolas Levrat/Yves Fricker (Hrsg.): Le Rapport du Conseil fédéral sur l'intégration européenne - Une évaluation interdisciplinaire; Collection Euryopa, Institut européen, Université de Genève, Genf 2000, S. 10-17. 
nannt, nach der so genannten ,Zauberformel ${ }^{6}{ }^{8}$ ist teilweise durch die direkte Demokratie mitbedingt. Die ausgeklügelten Gleichgewichte und Berücksichtigungen von Minderheitsrechten im Bundesrat und dem gesamten politischen System würden stark erschüttert durch die Beteiligung der Bundesregierung am europäischen Entscheidungsprozess und die Annahme und Umsetzung des Gemeinschaftsrechts ohne die Möglichkeit, mit Referenden zu drohen, um damit Sonderrechte und die Beachtung von verschiedensten regionalen Eigenarten herausschinden zu können. Vor einem EU-Beitritt benötigt die Schweiz mindestens eine Reform des Regierungssystems. Viertens sind die direkte Demokratie und die damit einhergehende politische Kultur zum wichtigsten Identitätselement des Landes geworden, das keine gemeinsame Sprache, Kultur oder Religion zusammenhält. Das Element der gemeinsamen politischen Kultur erscheint umso vordringlicher als die anderen traditionellen Integrationsfaktoren ihre Wichtigkeit verlieren. Mit dem Wegfall eines äußeren Feindes und der Bedrohung löst sich die nationale Klammer. Die Armee verliert kontinuierlich an Einfluss, und auch die Neutralität ist unwichtiger als früher. Schließlich treten die kulturellen Unterschiede im Zuge der Globalisierung verstärkt in den Vordergrund. Zum Beispiel ziehen heute die Sprachgruppen das Erlernen von Englisch dem einer zweiten Landessprache vor.

In einem Land, dem es an die Gemeinsamkeit fördernden Projekten mangelt und das auch nur sehr begrenzt über einen nationalen, öffentlichen Raum mit gemeinsamen Themen und Symbolen verfügt, sind die das politische Leben prägenden Volksabstimmungen praktisch die einzigen Momente, in denen sich die verschiedenen Volksteile begegnen. Zu guter Letzt ist die direkte Demokratie ein bewährtes Mittel, um die Minderheiten zu berücksichtigen und sie in das politische System einzubinden.

\section{Wirtschaftliche Besonderheiten der Schweiz}

Die Struktur der Schweizer Wirtschaft gleicht stark derjenigen der anderen westeuropäischen Länder. Sie ist auch hervorragend in die Weltwirtschaft integriert. Auf den ersten Blick scheint es keine wirtschaftlichen Hindernisse für einen EU-Beitritt zu geben. Bei näherer Betrachtung werden aber erhebliche wirtschaftspolitische Gründe gegen einen Beitritt sichtbar. So ist die Schweiz, von Norwegen vielleicht abgesehen, das einzige westeuropäische Land, in dem sich die Wirtschaftsverbände seit jeher überwiegend gegen eine EU-Mitgliedschaft aussprechen. ${ }^{9}$

Von den wirtschaftsrechtlichen Rahmenbedingungen der Schweiz, die sich von denen in der Union unterscheiden, profitieren wichtige international tätige Wirtschaftsbereiche. Die multinationalen Unternehmen, Banken und Versicherungen haben die Schweiz zu einem für sie sicheren Hafen von ,Spezial- und Sonderregelungen“ gemacht. Mit einem EU-Beitritt befürchten sie, in einen Strudel von interventionistischen Gemeinschafsnormen zu geraten, welche gerade diese für sie maßgeschneiderten Sonderregelungen hinwegfegen könnten. Auch besonders geschützte, kartellisierte und subventionierte Wirtschaftssektoren, wie zum Beispiel die Landwirtschaft, wollen ihre Privilegien bewahren. Die abgeschotteten Teile der Binnenwirtschaft haben zu Recht Angst vor der gemeinsamen Landwirtschaftspolitik, dem europäischen Wettbewerbsrecht und dem mit dem EU-Beitritt einhergehenden Wegfall der nichttarifären Hemmnisse. Ihre Interessenvertreter, unterstützt von denjenigen der off shore-Wirtschaftssektoren, benutzen die Hürden der direkten Demokratie, um ihre Pfründen zu bewahren.

8 Seit 2002 zwei Sozialdemokraten, zwei Freisinnige, zwei Vertreter der Nationalkonservativen, ein Christdemokrat.

9 Der Schweizer Arbeitgeberverband hat aber andere Integrationsformen unterstützt wie zum Beispiel das Freizügigkeitsabkommen von 1973 und die Bilateralen Verträge von 2002 und nun wieder jene von 2004. Er war auch für einen Beitritt der Schweiz zum EWR. Jedoch haben interne Zwistigkeiten in den Wirtschaftsverbänden erheblich zur Abweisung des EWR in der Volksabstimmung von 1992 beigetragen. 
Schließlich ist das schweizerische Bruttoinlandsprodukt das höchste in Europa trotz der relativen Erosion dieses komparativen Vorteils. Auch die unteren sozialen Bevölkerungsschichten profitieren vom allgemeinen Wohlstand. Sie befürchten eine Verschlechterung ihrer Lebensumstände im Falle des EU-Beitritts der Schweiz. Insgesamt hat die schweizerische Zurückhaltung gegenüber einer Mitgliedschaft in der Union somit nicht nur identitätsbezogene und politische sondern auch wichtige wirtschaftliche Gründe.

\section{Die zweiten bilateralen Abkommen - ein neuer Schritt auf dem dritten Weg}

\section{Rückblick auf die Entscheidung für den Bilateralismus}

Nach der negativ ausgefallenen Referendumsabstimmung über einen Beitritt der Schweiz zum EWR Ende 1992 entschied sich der Bundesrat für bilaterale Verhandlungen mit der Europäischen Union. Sie führten zum Abschluss der ersten sieben bilateralen Abkommen, die 2002 in Kraft getreten sind. Diese Verträge umfassen die Personenfreizügigkeit, den erleichterten Transit des Schwerverkehrs, den freien Luftverkehr, die Öffnung des kommunalen öffentlichen Beschaffungswesens, die Beteiligung der Schweiz an den europäischen Forschungsprogrammen, die Erleichterung des Handels mit Agrarprodukten und die Aufhebung der meisten seit dem in den 1970er Jahren abgeschlossenen Freihandelsabkommen noch verbliebenen technischen Handelshemmnisse. ${ }^{10}$

Wie ist zu erklären, dass die Schweizer die ersten bilateralen Abkommen mit einer eindeutigen Mehrheit von 67,2 Prozent der Stimmen und nur zwei abweisenden Kantonen angenommen haben, während sie den EWR stimmenmäßig knapp, auf der Ebene der Gliedstaaten aber sehr klar, verworfen hatten ${ }^{11}$ Unseres Erachtens sind dafür folgende Elemente ausschlaggebend: Erstens ist im Jahre 2000 der politische Kontext in Europa entspannter als 1992. Damals, kurz nach der deutschen Wiedervereinigung befürchteten viele Schweizer ein Wiedererstarken des deutschen Kolosses, der versuche, sich Europa nun friedlich und wirtschaftlich einzuverleiben. Zudem vermittelten der eben angenommene und die Europäische Union begründende Maastrichter Vertrag sowie die Referenden über ihn in Frankreich und Dänemark so manchem Schweizer den Eindruck, es werde gegen den Willen der Völker ein europäischer Bundesstaat errichtet. Zweitens hat die Schweizer Regierung seit der Verwirrung vor der EWR-Abstimmung konsequent zwischen der auf sektoriellen Abkommen beruhenden Teilintegration und einer EU-Mitgliedschaft unterschieden. Drittens ist die Souveränität der Schweiz mit den bilateralen Abkommen besser geschützt als im EWR. Die bilateralen Übereinkommen enthalten keine supranationalen Elemente und sie bedingen auch keine automatische Übernahme des sich laufend fortentwickelnden Gemeinschaftsrechts. Auch dieser Unterschied hat die an der formellen Souveränität des Landes hängen-

10 René Schwok/Nicolas Levrat: Switzerland's Relations with the EU after the Adoption of the Seven Bilateral Agreements, European Foreign Affairs Review, Vol. 6, No. 3, Autumn 2001, S. 335-354; René Schwok: Les accords sectoriels Union européenne-Suisse: une nouvelle forme de flexibilité dans la dimension extérieure de l'UE, Revue du Marché Commun et de l'Union européenne, Nr. 432, Oktober 1999; S. 613-619; Daniel Felder/ Christine Kaddous (Hrsg.): Bilaterale Abkommen Schweiz-EU: Erste Analysen, Basel/Genf/München/Brüssel 2001 und Daniel Thürer/Rolf H. Weber/Roger Zäch: Bilaterale Verträge Schweiz - EG: Ein Handbuch, Zürich 2002.

11 Etwas übertreibend und vereinfachend ausgedrückt, ist sowohl 1992 wie auch wieder 2000 der/die typische Europa-Gegner(in) ein(e) Deutschschweizer(in) oder italienischsprachige(r) Tessiner(in) ländlicher Herkunft, am Ende des zweiten oder im dritten Lebensalters stehend, mit einem mittelmäßigen Bildungsstand und somit auch nur über ein knappes oder einfaches wirtschaftliches Einkommen verfügend, öfter weiblich als männlich und schließlich Parteimitglied oder Sympathisant(in) der Schweizerischen Volkspartei. 
den Schweizer beruhigt. ${ }^{12}$ Viertens haben sich der Bundesrat, die Kantonsregierungen, die Wirtschaftsverbände und die Gewerkschaften anders als 1992 vorbehaltlos hinter die bilateralen Abkommen gestellt.

\section{Übersicht über die zweiten bilateralen Abkommen}

Am 26. Oktober 2004 haben die Europäische Union und die Schweiz eine zweite Serie bilateraler Abkommen unterzeichnet. In der Schweiz sind die Verträge zum Teil sehr umstritten. Das Übereinkommen über die Assoziierung mit dem Schengen-Raum ist, nach Erreichung der für ein Referendum notwendigen Unterschriftenzahl, am 5. Juni 2005 zur Volksabstimmung unterbreitet worden. Das Abkommen wurde mit 54,6 Prozent der Stimmen angenommen. Die Mehrheit der Kantone wurde aber nicht erreicht. Da die Verfassung für die Annahme dieses Abkommens jedoch nur das Volksmehr vorschreibt, wird es voraussichtlich 2008 in Kraft treten. Die Abkommen umfassen neun Bereiche (siehe Abbildung 1).

\section{Abbildung 1: Die neun bilateralen Abkommen II zwischen der EU und der Schweiz}

- Polizeiliche und justizielle Zusammenarbeit, Asyl und Migration (Schengen/Dublin). Die Schweiz beteiligt sich am Schengen/Dublin-System gemäß dem schon für Norwegen und Island bestehenden Modell. Die Schweiz kann ihre Grenzkrontrollen aufrechterhalten. Zudem erhält sie Garantien bezüglich ihres Bankgeheimnisses.

- Zinsbesteuerung. Die Schweiz besteuert die Spareinkommen von in der EU sesshaften Bürgern. Ihr Bankgeheimnis bleibt gewahrt.

- Betrugsbekämpfung. Die Schweiz hilft der EU im Kampf gegen den Zollbetrug und die Umgehung von indirekten Steuern. Auch diesbezüglich erhält sie Garantien in Sachen Bankgeheimnis.

- Verarbeitete Landwirtschaftsprodukte. Senkung der Zölle betreffend die verarbeiteten Landwirtschaftsprodukte (z. B. Schokolade, Kekse, Suppen, wasserlöslicher Kaffee).

- Statistik. Das bilaterale Abkommen über die Zusammenarbeit im Bereich der Statistik regelt die progressive Harmonisierung der statistischen Datenerhebung zwischen der Schweiz und der EU.

- Renten. Die Schweiz verzichtet auf die Besteuerung der auf ihrem Gebiet sesshaften pensionierten Beamten der EU. Zurzeit sind nur ungefähr 50 Personen betroffen.

- Umwelt. Die Schweiz wird Mitglied der Europäischen Umweltagentur.

- Media. Teilnahme der Schweiz am europäischen Programm MEDIA zur Förderung der Entwicklung und des Vertriebs gemeinschaftlicher audiovisueller Werke und der Ausbildung von Berufsangehörigen der audiovisuellen Programmindustrie.

- Bildung, Berufsbildung, Jugend. Teilnahme der Schweiz ab 2007 an den europäischen Jugendförderprogrammen SOKRATES (allgemeine Bildung), LEONARDO DA VINCI (Berufsbildung) und JUGEND (außerschulische Jugendarbeit).

Sechs Dossiers waren unproblematisch. Drei gaben Anlass zu harten diplomatischen Verhandlungen: die Assoziierung mit ,Schengen', die Betrugsbekämpfung und die Besteuerung von Zinserträgen. In unserem Beitrag stellen wir die drei umstrittenen Abkommen vor. Wir beschreiben kurz ihren Inhalt und zeigen ihre institutionellen Besonderheiten auf. Darauf aufbauend folgt jeweils eine politische Beurteilung der Verhandlungsresultate. Es wird ge-

12 Dieser rechtlich-formelle Umstand ändert nichts an der Tatsache, dass die Schweiz sich seit langem und immer stärker autonom an die Entwicklung der wirtschaftlichen Gepflogenheiten und rechtlichen Rahmenbedingungen des gemeinsamen europäischen Marktes anpassen muss. 
zeigt, wie die Schweiz auch mit diesen zweiten bilateralen Übereinkommen ihren dritten Weg zwischen EU-Mitgliedschaft und Isolation vorderhand erfolgreich weiter beschreitet.

Das Abkommen zur polizeilichen und justiziellen Zusammenarbeit, Asyl und Migration (Schengen/Dublin $)^{13}$

Das Abkommen assoziiert die Schweiz an den so genannten Schengen/Dublin-Acquis. Zur Erinnerung: Schengen erleichtert die Personenfreizügigkeit dank dem Verzicht auf die systematische Personenkontrolle beim EU-internen Grenzübertritt. Zudem verbessert der Vertrag die grenzüberschreitende Zusammenarbeit der Polizei- und Justizbehörden in ihrem Kampf gegen das internationale Verbrechen und den Terrorismus.

Die Schweiz wird Zugang zum Schengener Informationssystem (SIS) erhalten. Es handelt sich um eine Datenbank über polizeilich gesuchte Personen und Sachen. Das SIS hat sich vor allem bei der Bekämpfung von Schmuggel, Grenzkontrollumgehung, Waffenhandel und Drogenkriminalität als sehr nützlich erwiesen.

Das Dublin-Abkommen regelt die Zuständigkeit der Mitgliedstaaten zur Behandlung der im EU-Raum gestellten Asylgesuche. Dank der elektronischen Datenbank Eurodac, in der die Fingerabdrücke der Asylsuchenden gespeichert sind, kann eine Person, welche mehrere Gesuche gestellt hat, besser identifiziert und gegebenenfalls in den Erstgesuchsstaat zurückgeführt werden.

Inhalt und institutionelle Besonderheiten des Abkommens. Das Abkommen sieht vor, dass die Schweiz im Schengen/Dublin-Raum den gleichen Status erhält wie Norwegen und Island. Wie diese schon angeschlossenen Nicht-EU-Staaten wird sie an der politischen Weiterentwicklung des Schengen/Dublin-Rechtsbestands teilnehmen können (,decision shaping ${ }^{6}$ ), dabei aber kein formelles Mitentscheidungsrecht haben. Sie wird bei der Behandlung der sie betreffenden Fragen, zum Beispiel bei Abänderungen des Asyl- und Visumrechts, ihre Interessen in den Entscheidungsprozess einbringen können. Wie im Falle des EWR wird die Schweiz ohne Stimmrecht aber ansonsten gleichberechtigt an der zukünftigen Entwicklung des Schengen-Acquis mitwirken können. Zudem wird sie vollen Zugang zu neuen Instrumenten wie dem Schengen Informationssystem II (SIS II) erhalten.

Bei jeder Änderung des Schengen/Dublin-Rechts wird die Schweiz autonom und souverän entscheiden können, ob sie den neuen Rechtsakt übernehmen und umsetzen will. Dafür hat sie eine Übergangsfrist von bis zu zwei Jahren ausgehandelt. Die Anpassung des schweizerischen Rechts an den evolutiven Schengen/Dublin-Acquis erfolgt nicht automatisch, sondern wird jedes Mal dem schweizerischen Gesetzgeber vorgelegt werden. Wenn die Schweiz während des Rechtssetzungsprozesses ankündigt, eine neue Rechtsnorm nicht übernehmen zu wollen, und mit den anderen Mitgliedsstaaten kein Kompromiss gefunden werden kann, steht es ihr letztlich frei, das Abkommen zu kündigen. Dieses Verfahren wird insoweit locker gehandhabt werden, als die Schweiz in wichtigen Fällen kurzfristig eine Ministerzusammenkunft einberufen kann und alternative Vorschläge unterbreiten darf.

13 Die wichtigsten Dokumente hierzu sind zwei Rahmenabkommen: Abkommen zwischen der Schweizerischen Eidgenossenschaft, der Europäischen Union und der Europäischen Gemeinschaft über die Assoziierung dieses Staates bei der Umsetzung, Anwendung und Entwicklung des Schengen-Besitzstands, http://www.europa. admin.ch/nbv/off/abkommen/d/schengen_1.pdf (letzter Zugriff: 14.6.2005) und Abkommen zwischen der Schweizerischen Eidgenossenschaft und der Europäsichen Gemeinschaft über die Kriterien und Verfahren zur Bestimmung des zuständigen Staates für die Prüfung eines in einem Mitgliedstaat oder in der Schweiz gestellten Asylantrags ,http://www.europa.admin.ch/nbv/off/abkommen/d/dublin_1.pdf (letzter Zugriff: 14.6.2005). Vgl. allgemein auch: Integrationsbüro EDA/EVD, Bilaterale Abkommen II, Schweiz Europäische Union: Fact Sheets, Bern 2004, http://www.europa.admin.ch/nbv/expl/factsheets/d/index.htm (letzter Zugriff: 14.6.2005). 
Das Abkommen Schweiz-EU über den Schengen-Acquis beinhaltet auch die Pflicht zur Rechtshilfe. Hierzu hat die Schweiz eine Sonderlösung ausgehandelt, welche die Bewahrung des Bankgeheimnisses sichert. Sollte eines Tages die Entwicklung des Schengen-Acquis die Rechtshilfegewährung bei Steuerhinterziehung verlangen, ist vorgesehen, dass die Schweiz davon ausgenommen bleibt, ohne dass sie auf die Teilnahme am Schengener Sicherheitsraum verzichten muss.

Politische Bewertung. Für die Union ist das Abkommen wichtig, weil sich die Schweiz im geografischen Herzen des Schengen/Dublin-Dispositivs befindet, ohne bisher darin eingebunden zu sein. Seit vielen Jahren haben die Zollbehörden der Nachbarstaaten der Schweiz grosse Mühe, den von ihr ausgehenden Landgrenzverkehr, abgesehen von einigen wenigen Hauptachsen, zu kontrollieren.

Die Integration der Schweiz ins EU-Sicherheitsdispositiv wird es der Union ermöglichen, koordinierter gegen das organisierte Verbrechen vorzugehen, die illegalen Geldflüsse vermehrt einzudämmen, die Lasten der Asylpolitiken besser zu verteilen und die innereuropäische Mobilität zu erleichtern, letzteres insbesondere zu Gunsten von Tausenden Grenzgängern, die täglich in die Schweiz zur Arbeit kommen.

Aus Sicht der Europäischen Union kann man hervorheben, dass es ihr nicht nur gelungen ist, die Schweiz zur Übernahme ihrer Rechtsinstrumente zu bewegen. Auch deren Weiterentwicklung wird im Prinzip akzeptiert, ohne dass die Eidgenossen darüber formell mitentscheiden können. Es ist der Union gelungen, die integrationsscheuen Schweizer in die zukünftige Schengen-Rechtsentwicklung einzubinden und zugleich die im Entscheidungsprozess eventuell durch sie entstehende Blockadegefahr auszuschalten. Zudem hat die Union eine Anpassung des schweizerischen Bankgeheimnisses erreicht und damit ihren Handlungsspielraum im Kampf gegen das organisierte Verbrechen vergrößert.

Für die Schweiz ist die Bilanz ebenfalls positiv. Ungefähr 700.000 Personen, 300.000 Personenwagen und 20.000 Lastwagen überqueren täglich ihre Landesgrenzen. Das Grenzwachtkorps ist nicht in der Lage, dieses Ausmaß an Verkehr systematisch zu kontrollieren. Mehr als 97 Prozent der Personen überschreiten ungeprüft die Schweizer Grenze. In Anbetracht dessen ändert die offizielle Aufhebung der Personenkontrolle nicht viel an der bestehenden Lage. Umso nützlicher für die Schweiz ist die Teilnahme am Schengener Sicherheitssystem. Der volle und freie Zugriff auf die Datenbanken des SIS und des Eurodac wird ihre Schlagkraft in der Verbrechensbekämpfung stärken. Die Schweiz wird allgemein besser in die europäische Polizei- und Justizzusammenarbeit eingebunden.

Die Assoziierung an das Dubliner Abkommen bringt auch wirtschaftliche Vorteile für die Schweiz. Sie wird mit der Europäischen Union eine gemeinsame Visumpolitik bestimmen können. In Zukunft werden Reisende aus Asien, Afrika und Lateinamerika, welche schon über ein Eintrittsvisum in den Schengen-Raum verfügen, kein Zweitvisum für die Schweiz mehr beantragen müssen. In Zeiten einer globalisierten und in einem harten Wettbewerb stehenden Fremdenverkehrsindustrie ist jede Erleichterung des Grenzübertritts ein wertvoller Gewinn für den Schweizer Tourismus.

Wir unterstreichen jedoch, dass die Assoziierung der Schweiz an das Schengener Abkommen an der aktuellen Situation an ihrer Grenze sehr wahrscheinlich nicht viel ändern wird, im Unterschied zu einem Schengen-Beitritt als EU-Mitgliedstaat. Die Europäische Union ist eine Zollunion. Die Schweiz hingegen befindet sich mit ihr nur in einer Freihandelszone. Auch wenn der Freihandel für Industriegüter gewährleistet ist, darf die Schweiz deren Herkunft überprüfen, da sie nicht dem gemeinsamen Zolltarif unterliegt. Zudem ist das Landwirtschaftsabkommen nur partiell. Die Schweiz ist auch nicht in die europäische Zone für 
indirekte Steuern integriert. Aus all diesen Gründen können die Schweizer Grenzbehörden weiterhin Waren- und Fiskalkontrollen durchführen und dabei auch Personen untersuchen, die ihnen verdächtig erscheinen.

Abschließend ist auch die Zufriedenheit der schweizerischen Banken hervorzuheben. Mit dem Schengener Abkommen erhält die Eidgenossenschaft die völkerrechtliche Anerkennung ihres Bankgeheimnisses in Sachen direkte Besteuerung, und dies auch im Falle der rechtlichen Weiterentwicklung des Schengen-Acquis.

Kurzanalyse der Volksabstimmung vom 5. Juni 2005. Wie erwähnt hat eine deutliche Mehrheit von 54,6 Prozent der Stimmbürger ${ }^{14}$ das Schengen/Dublin-Abkommen gutgeheißen. ${ }^{15}$ Die Wahlbeteiligung lag mit 55,9 Prozent etwa 10 Prozent über dem Durchschnitt der letzten Jahre. Das Resultat ist also auch in dieser Hinsicht legitim. In jüngster Zeit haben nur bei der Uno-Beitrittsabstimmung (im März 2002) so viele Bürger von ihrem Stimmrecht Gebrauch gemacht.

Trotz der positiven Aspekte hatte die national-konservative politische Rechte, die Schweizerische Volkspartei (SVP) und die Aktion für eine unabhängige und neutrale Schweiz (AUNS) das Referendum gegen die Schengen/Dublin-Assoziierung ergriffen. Seit den Bundesparlamentswahlen 2003 ist die SVP die wählerstärkste Partei des Landes. Sie hat rund ein Viertel der Wählerschaft hinter sich geschart und verfügt über zwei Sitze in der Bundesregierung. Ihr charismatischer Anführer, Christoph Blocher, hält einen dieser Sitze als Chef des Justiz- und Polizeidepartements. Durch die Fügung der Politik ist er als solcher für das Schengen/Dublin-Dossier zuständig geworden. Dieser Umstand hatte während des Abstimmungskampfes zu sehr ungewöhnlichen, wenn nicht gar einmaligen Polemiken geführt. In der Tat hatte Bundesrat Blocher in seiner Ministerfunktion und Sachzuständigkeit, aber im Gegensatz zu seiner persönlichen Überzeugung die Schengen/Dublin bejahende Regierungsposition vor der Abstimmung kollegial in der Öffentlichkeit mitvertreten müssen.

Die Gegenargumente der SVP und der AUNS nährten sich von der Angst eines Souveränitätsverlustes der Schweiz. Sie behaupteten, das Land unterstelle sich ausländischen Richtern. Sie befürchteten nicht nur, dass die Grenzkontrollen verschwinden könnten, sie sagten auch, dass mit Schengen/Dublin die Symbolik der Grenze verwässert würde. Manche unter ihnen sind sogar der Überzeugung, die Assoziierung an Schengen/Dublin verstricke die Schweiz in ein Integrationsnetz, aus dem sich das Land nicht mehr herauswinden könne und so den EU-Vollbeitritt in absehbarer Zeit unausweichlich mache. Tatsächlich ist die Grenze ein Garant für Identität und Eigenständigkeit. Die staatliche Grenzkontrolle ist der konkrete und symbolisch aufgeladene Ausdruck der nationalen Unabhängigkeit. Unter diesem Gesichtspunkt ist die Abweisung von Schengen/Dublin mit der ihr zu Grunde liegenden Öffnungsphilosophie und gewollten institutionellen Zusammenarbeit unter sich nicht mehr fremden, sondern freundschaftlich verbundenen Partnern durch die national-konservativen

14 Es stimmten aber nur 12 Kantone dafür und 13 dagegen. Für einen EU-Beitritt wäre die doppelte Mehrheit der Bürger und der Kantone erforderlich. Dies entspricht ungefähr einem Stimmenmehr von 56 Prozent.

15 Die Abstimmung betreffend die Schengen/Dublin-Assoziierung darf nicht mit der Volksabstimmung vom 25. September 2005 verwechselt werden. Dann werden die Schweizer über die Ausdehnung des Personenfreizügigkeitsabkommens auf die zehn neuen EU-Mitgliedstaaten zu befinden haben. Sollten die Schweizer diese Ausdehnung ablehnen, wäre es aus der Sicht der Union logisch, die gesamten bilateralen Abkommen mit der Schweiz zu kündigen. Sie könnte wohl schwerlich annehmen, dass in der Beziehung mit einem Drittstaat die für sie zentrale Personenfreizügigkeit nicht für alle Mitgliedstaaten und ihre Bürger gelten könnte. So hat EUAußenkommissarin Benita Ferrero-Waldner am 6. Juni, einen Tag nach der Abstimmung zu Schengen/Dublin, entgegen der juristischen Unabhängigkeit zwischen Schengen/Dublin und der Personenfreizügigkeit, den politischen Link zwischen den beiden Problemkreisen geschlagen und die Schweiz darauf hingewiesen, dass ohne die Annahme der Freizügigkeitserweiterung die EU das Schengen/Dublin-Abkommen vielleicht nicht ratifizieren wird (Quelle: Swissinfo, 6.6.2005). 
und manchmal fremdenfeindlichen Bewegungen folgerichtig. Es ist aber doch bemerkenswert, dass gerade diejenigen Kreise, welche die Bewahrung und Stärkung des Schweizertums auf ihre Fahnen geschrieben haben und ständig für eine strenge Asylpolitik und den Kampf gegen jegliches Verbrechen plädieren, die vorgeschlagenen und bewährten Handlungsmöglichkeiten des Schengen/Dublin-Systems ausschlugen, nur weil sie europaweit organisiert sind.

Im Gegensatz dazu hatte sich die überwiegende Mehrheit der politischen Parteien, der Arbeitgeber- und Arbeitnehmerorganisationen zusammengerafft, um die Schweiz zum Beitritt zu Schengen/Dublin zu bewegen. Schon Monate vor der Volksabstimmung erschienen in der Presse Aufrufe zur Annahme des Abkommens. Die liberal-demokratische Rechte und die Banken unterstützten das Schengen/Dublin-Abkommen vor allem aus zwei Gründen: Erstens bringt der Vertrag rechtliche Garantien für das Bankgeheimnis. Zweitens wollten sie einen Dominoeffekt mit Blick auf die anderen acht Abkommen, darunter das für sie wichtige Zinsbesteuerungsabkommen verhindern. Auch wenn die zweiten bilateralen Abkommen rechtlich nicht miteinander verknüpft sind, hätte im Falle der Verwerfung des Schengen/Dublin Abkommens durch die Schweizer Stimmbürger ein EU-Mitgliedstaat vielleicht versucht sein können, im EG-internen Ratifizierungsprozess das gesamte Paket infrage zu stellen.

Der finanzstarke Einsatz der Befürworter einer Assoziierung an das Sicherheitssystem von Schengen und Dublin hat sich ausgezahlt. Die großen deutschschweizerischen Städte Zürich, Basel und Bern sowie die französischsprachige Westschweiz haben die Vorlage mit klaren Mehrheiten zwischen 57 Prozent und bis zu 70 Prozent angenommen. Die Allianz der Großstädte mit der in außenpolitischen Fragen traditionell offenen Westschweiz konnte die konservativen deutschsprachigen Landregionen und das italienischsprachige Tessin übertreffen.

Die Abstimmung vom 5. Juni 2005 ist geradezu ein Paradebeispiel der außenpolitischen und gesellschaftspolitischen Kluft, die seit mehr als einem Jahrzehnt sowohl die Städte von den ländlichen und alpinen Regionen der Zentral- und Ostschweiz als auch die Mehrheit der Deutschschweizer Kantone und des Tessins von der weltoffeneren, französischsprachigen Westschweiz trennt. Nur ein Zusammenschluss der Stadtbevölkerung mit der Romandie ist in der Lage, die konservative Hürde der Land- und Bergkantone zu überspringen. Abstimmungen, die der doppelten Mehrheit der Stimmbevölkerung und der Kantone bedürfen, wie zum Beispiel Verfassungsänderungen oder der Beitritt zu einer supranationalen Organisation wie der Europäischen Union, riskieren momentan, am vom Föderalismus unwillentlich geförderten konservativen Deutschschweizer alpinen Block zu zerschellen.

\section{Das Zinsbesteuerungsabkommen ${ }^{16}$}

Der Kern des Abkommens ist die Verpflichtung der Schweiz, ab 2011 Sparzinserträge von natürlichen Personen, welche ihr Steuerdomizil in der Union haben, mit einer Steuer von 35 Prozent zu belegen. 75 Prozent des erhobenen Geldes wird den Steuerbehörden der EUStaaten überwiesen werden. Im Gegenzug bewahrt die Schweiz ihr Bankgeheimnis. Nach den Ratifizierungen soll der Vertrag am 1. Juli 2005 in Kraft treten.

Inhalt und institutionnelle Besonderheiten des Abkommens. Seit Ende der 1980er Jahre versuchen die EU-Staaten ihre Bürger davon abzuhalten, ihr Geld in anderen

16 Abkommen zwischen der Schweizerischen Eidgenossenschaft und der Europäischen Gemeinschaft über Regelungen, die den in der Richtlinie 2003/48/EG des Rates im Bereich der Besteuerung von Zinserträgen festgelegten Regelungen gleichwertig sind, http://www.europa.admin.ch/nbv/off/abkommen/d/tax_1.pdf (letzter Zugriff: 14.6.2005) 
Ländern anzulegen, um so der Besteuerung ihrer Spareinkommen zu entkommen. Zur Verhinderung der Steuerflucht haben die Europäische Kommission und die Mehrzahl der Mitgliedstaaten vorgeschlagen, einen automatischen Informationsaustausch zwischen den nationalen Steuerbehörden einzurichten. Drei Mitgliedstaaten, Luxemburg, Belgien und Österreich, wollten jedoch für den Fall der Steuerflucht ihr Bankgeheimnis nicht lüften. Auch die Schweiz, Andorra, San Marino und Liechtenstein sowie die von Großbritannien und den Niederlanden abhängigen Steuerparadiese zeigten eine abweisende Haltung. Die Europäische Union war damit gezwungen, diese Drittstaaten, insbesondere die Schweiz, in ihre Bemühungen einzubeziehen. Es galt auch Luxemburg, Belgien und Österreich, welche die Abwanderung des von ihnen verwalteten Geldes in die Schweiz und in andere sicherere Bankhäfen befürchteten, zu besänftigen und bei der Stange zu halten. Da in der Steuerpolitik EU-intern das Einstimmigkeitsprinzip gilt, können diese drei Staaten nämlich jegliche Entscheidung mit ihrem Veto verhindern. Um dieses Dilemma zu überwinden, fußte das erste europäische Richtlinienprojekt zur Ersparnisbesteuerung (1998) auf dem so genannten Koexistenzmodell. Die EU-Staaten hätten danach die Wahl zwischen dem automatischen Informationsaustausch (Bankgeheimnisaufhebung) und dem Steuerrückbehalt gehabt. Dieser Vorschlag wurde jedoch beim Treffen des Europäischen Rates in Feira im Juni 2000 grundsätzlich geändert. Die Staats- und Regierungschefs ließen plötzlich die Koexistenzidee fallen und verlangten wiederum die Einführung des automatischen Informationsaustausches unter der Bedingung, dass die Schweiz und die anderen europäischen Drittstaaten mitzögen. Um sich nicht mehr ihren EU-Partnern entgegen zu stellen und sich von ihren Druckversuchen zu befreien, hatten Luxemburg, Belgien und Österreich einen taktischen Schachzug vollzogen. Sie machten ihr Mitmachen vom Einlenken der Schweiz abhängig - im Wissen, dass die Eidgenossenschaft nicht dazu bereit sein würde. Paradoxerweise wurde damit einem Drittstaat ein Quasivetorecht in einer EU-internen Angelegenheit zugestanden. Indirekt übertrug man der Schweiz die Entscheidung über die Zukunft des EU-Richtlinienentwurfs zur Ersparnisbesteuerung. ${ }^{17}$ Es handelte sich um eine in der Integrationsgeschichte einmalige Konstellation!

In der Schweiz verursachte der EU-Gipfel in Feira ein kleines Psychodrama. ${ }^{18}$ Ein Einlenken bei der Frage des automatischen Informationsaustausches wäre dieser Aufgabe des Bankgeheimnisses in allen Fällen von Steuerflucht aus der Union gleichgekommen. In Anbetracht der helvetischen Unnachgiebigkeit, des Doppelspiels der drei genannten Mitgliedstaaten, mehrerer zweideutiger Stellungnahmen seitens Großbritanniens und letztlich auch des Unwillens der Vereinigten Staaten über die Problematik der Steuerflucht auf transatlantischer Ebene zu verhandeln, ist die Europäische Union schließlich de facto auf das Koexistenzmodell zurückgeschwenkt. Sie hat sich mit den Bankgeheimnissen abgefunden, die in den drei EU-Staaten Österreich, Belgien und Luxemburg, der Schweiz sowie den europäischen Mikrostaaten und in den von Großbritannien und den Niederlanden abhängigen Territorien gelten. Als Gegenleistung für die Beibehaltung des Bankgeheimnisses müssen die genannten Staaten und Territorien ein Quellensteuersystem für Sparzinseinnahmen einführen. Die Steuer soll im Endeffekt 35 Prozent des erwirtschafteten Zinses umfassen. In der Schweiz wird das den Zins gutschreibende Unternehmen oder Finanzinstitut verpflichtet,

17 Cristophe Bonte: Comprendre la logique politique de l'UE pour comprendre les négociations actuelles, in: René Schwok (Hrsg.): Place financière suisse, évasion fiscale et intégration européenne, Genève 2002, S. 5662.

18 René Schwok: „Typologie des différents scénarios“, in: Schwok (Hrsg.): Place financière suisse, S. 185-191. 
den Rückbehalt zu tätigen. Der Rückbehalt von 35 Prozent wird progressiv eingeführt. In den ersten drei Jahren wird er 15 Prozent, in den drei folgenden 20 Prozent und schließlich 35 Prozent umfassen.

Einige weitere Elemente des Abkommens verdienen Beachtung. In einem Memorandum ${ }^{19}$ haben sich die Schweiz und die EU-Staaten verpflichtet, auf Nachfrage gegenseitig Informationen betreffend ,Steuerbetrug und analoge Vergehen' auszutauschen. Diese Art von Verwaltungszusammenarbeit mit der Europäischen Union ist neu. Es ist jedoch zu beachten, dass in der Schweiz die Nichtangabe von Vermögenswerten nicht als Steuerbetrug gilt. Sie ist nur ein steuerrechtlich strafbares Versehen, jedoch kein strafrechtliches Delikt. Um auf Steuerbetrug schließen zu können, bedarf es der vorsätzlichen, böswilligen Widerrechtlichkeit wie zum Beispiel im Falle der Dokumentenfälschung. Diese subtile Unterscheidung wird von verschiedenen Ländern, von linken Politikern und von einigen kritischen Bankiers zurückgewiesen. Ebenfalls erwähnenswert ist der Artikel 15 des Abkommens, der für die Schweiz interessantes Element enthält. Er hebt die Quellensteuer für Zinszahlungen, Dividenden und Lizenzabgaben zwischen assoziierten Unternehmen auf. Damit werden Schweizer Mutterunternehmen, welche mit im EU-Raum etablierten Zweigunternehmen verbunden sind, im Vergleich zu ähnlichen europäischen Großunternehmen nicht mehr diskriminiert. Dies ist für international tätige Schweizer Gesellschaften und für die zahlreichen in der Schweiz niedergelassenen Holdinggesellschaften sehr wichtig. Das Steuerabkommen enthält schließlich auch Revisionsbestimmungen. Erst nachdem der Vertrag in Kraft getreten ist, kann eventuell eine Abänderung vorgenommen werden. Dies könnte frühestens nach 2013 eintreffen. Mit anderen Worten ist ein Systemwechsel zum automatischen Informationsaustausch bis dahin ausgeschlossen, und die Aufhebung des Bankgeheimnisses ist für längere Zeit vom Tisch.

Politische Bewertung. Für die Europäische Union ist das Zinsbesteuerungsabkommen ein Misserfolg. Sie hatte mit der anfänglich am Thema wenig interessierten Schweiz die Verhandlung gesucht. Die Resultate bleiben aber unter ihren Erwartungen. Die EU-Wunschvorstellungen waren erstens eine einheitliche Zinsbesteuerungspolitik zu entwickeln, zweitens den Druck auf die Drittstaaten und Steuerparadiese aufrechtzuerhalten, drittens die Steuerflucht zu verhindern und viertens verlorenes Geld zurückzuerhalten.

Das erste Ziel wurde verfehlt. Die drei EU-Mitgliedstaaten Luxemburg, Belgien und Österreich haben sich eine vielleicht dauerhafte Ausnahmesituation erstritten. Sie behalten ihr Bankgeheimnis, während die anderen zweiundzwanzig Staaten zum automatischen Informationsaustausch übertreten. Auch in anderer Hinsicht ist das Abkommen kein Erfolg. Der Zinsbesteuerungsvertrag mit der Schweiz blockt die Bestrebungen auf der OECD-Ebene abrupt ab, das Bankgeheimnis zu brechen. Anders ausgedrückt: Dieses Abkommen erkennt zum ersten Mal implizit das Bankgeheimnis im Bereich der Steuerflucht an. Das dritte Ziel wurde ebenfalls weitgehend verfehlt. In der Tat ist es sehr zweifelhaft, ob dieses Abkommen die Steuerflucht eindämmen wird. Der 35-prozentige Zinssatz ist dafür wahrscheinlich zu wenig abschreckend. Es ist zu erwarten, dass die schweizerischen, die luxemburgischen und die anderen Banken ihren Kunden Finanzinstrumente anbieten werden, mit denen sie diese Steuer umgehen können (Bankversicherungen, Ansiedlung des zahlenden Agenten außerhalb Europas, off-shore- und ,Briefkastengesellschaften“ und so weiter). Bezüglich des vierten EU-Bestrebens vermuten wir, dass wenig Geld in die Kassen der Mitgliederstaaten zu-

19 Das zwischen der Schweizerischen Eidgenossenschaft, der Europäischen Gemeinschaft und den EU-Mitgliedstaaten abgeschlossene Memorandum ist dem Zinsbesteuerungsabkommen beigelegt: http://www.europa.adm in.ch/nbv/off/abkommen/d/tax_1.pdf (letzter Zugriff: 16.6.2005). 
rückfließen wird. In Anbetracht der oben angeführten Gründe werden die meisten europäischen Bankkunden voraussichtlich Mittel finden, um den Rückbehalt von 35 Prozent ihrer Zinseinkünfte vermeiden zu können.

Schweizerischerseits hingegen ist das Verhandlungsresultat mit kaum verhohlenem Aufatmen aufgenommen worden. Das nach der anfänglich bedrohlichen Ausgangssituation erreichte Zinsbesteuerungsabkommen stellt einen Sieg dar. Das Resultat entspricht grosso modo den Wünschen des Bundesrates. ${ }^{20}$ Er hat es geschafft, dem Drängen auf die Abschaffung des Bankgeheimnisses in Sachen Steuerhinterziehung zu widerstehen. Er hat ebenfalls erreicht, dass kein anderes in diese Richtung gehendes Bestreben in den kommenden 15 Jahren zu erwarten ist. Letztlich werden die Schweizer Steuerbehörden voraussichtlich nicht so viel Geld an ihre EU-Partner zu erstatten haben, wie diese erhofften.

Dies alles erklärt, warum der Schweizer Finanzplatz, die Wirtschaftsverbände, der Bundesrat und die bürgerlichen Parteien, außer der SVP, sich für die Annahme des Schengen-Referendums am 5. Juni 2005 und somit für die reibungslose Annahme des gesamten Pakets der zweiten bilateralen Abkommen eingesetzt hatten. Desgleichen werden sie für die Annahme der Volksabstimmung vom 25. September 2005 betreffend die Ausdehnung des Übereinkommens zur Personenfreizügigkeit auf die neuen EU-Mitgliedstaaten kämpfen. ${ }^{21}$ In der Tat geht es im Herbst um die definitive Absicherung des gesamten Systems der ersten und zweiten bilateralen Abkommen mit seinen, wie dargestellt, für die Schweiz sehr wichtigen wirtschaftspolitischen Vorzügen.

\section{Das Betrugsbekämpfungsabkommen ${ }^{22}$}

Das Betrugsbekämpfungsabkommen strebt eine intensivere Zusammenarbeit zwischen der Schweiz und der Europäischen Gemeinschaft im Kampf gegen Schmuggel und andere Deliktformen im Bereich indirekte Steuern (Zoll, Mehrwertsteuer, Verbrauchssteuer), bei Subventionen sowie im öffentlichen Beschaffungswesen an. Die Union hatte der Schweiz vorgeworfen, als Finanzdrehpunkt für Schmuggel und andere Delikte im Zusammenhang mit dem internationalen Warenhandel zu fungieren. Vor allem hatte die Union wiederholt auf die Rolle des Bankgeheimnisses in diesem Kontext hingewiesen und behauptet, es leiste solchen Delikten Vorschub.

Das Zollbetrugsabkommen soll dazu beitragen, die Amts- und Rechtshilfe, das heißt die internationale Zusammenarbeit von Verwaltungs- und Justizbehörden zwischen der Schweiz und den EU-Staaten griffiger zu gestalten und den Informationsaustausch zwischen den Vertragsstaaten zu verbessern. Der Anwendungsbereich des Vertrags umfasst Delikte in den Bereichen indirekte Steuern und Subventionen sowie im öffentlichen Beschaffungswesen. $\mathrm{Zu}$ den indirekten Steuern zählen Zollabgaben, Mehrwertsteuern, besondere Verbrauchssteuern auf Alkohol, Tabakwaren, Mineralöl und so weiter. Das Abkommen klammert die direkten Steuern aus.

20 Bundesrat, ,Eröffnung der Verhandlungen zur Zinsbesteuerung“, EFD-Medienmitteilung vom 18.7.2002, Eidgenössisches Finanzdepartement, http://www.europa.admin.ch/nbv/medien/2002/d/pm_020618.pdf (letzter Zugriff: 14.6.2005).

21 Wie schon erwähnt, ist dies eine für die Europäische Union sehr wichtige Anpassung der ersten bilateralen Verträge. Gemäß einer Meinungsumfrage vom 12.6.2005 wollen zurzeit nur 35 Prozent der Befragten der Erweiterung zustimmen. 44 Prozent der befragten Personen würden sie ablehnen und 21 Prozent haben ihre Meinung noch nicht gefasst (Quelle: Zeitung Le Matin und Swissinfo vom 12. Juni 2005).

22 Abkommen über die Zusammenarbeit zwischen der Schweizerischen Eidgenossenschaft einerseits und der Europäischen Gemeinschaft und ihren Mitgliedstaaten andererseits zur Bekämpfung von Betrug und sonstigen rechtswidrigen Handlungen, die ihre finanziellen Interessen beeinträchtigen, http://www.europa.admin.ch/nbv/ off/abkommen/d/dog_1.pdf (letzter Zugriff: 14.6.2005). 
Inhalt und institutionelle Besonderheiten des Abkommens. Gemäß dem Abkommen verpflichtet sich die Schweiz, den EG-Behörden und denjenigen der Mitgliedstaaten, bei rechtswidrigen Handlungen in den Bereichen indirekte Steuern, Subventionen und öffentliches Beschaffungswesen in der gegenseitigen Zusammenarbeit die gleiche Behandlung zu gewähren, wie sie nach schweizerischem Recht und Praxis in einem landesinternen Verfahren erbracht werden muss. Zwangsmaßnahmen wie zum Beispiel Hausdurchsuchungen, Beschlagnahmungen und Einsichtnahme in Bankkonten werden fortan nicht nur im Rahmen der Rechtshilfe gewährt, sondern neu unter gewissen Umständen und Bedingungen auch schon in der Amtshilfe. Im Fall von Geldwäsche im Bereich der indirekten Steuern wird die Schweiz Rechtshilfe leisten, wenn das Geld aus einem Delikt stammt, das nach schweizerischem Recht, wenn es in der Schweiz begangen würde, als Abgabebetrug oder gewerbsmässiger Schmuggel behandelt würde.

Politische Bewertung. Das Abkommen wird der Europäischen Union in Zukunft helfen, den Zigarettenschmuggel und andere Betrugsarten besser zu bekämpfen und so beträchtliche Steuerausfälle zu vermeiden. Die Zusammenarbeit wird auch auf die Geldwäsche ausgedehnt. Für die Schweiz ist das Abkommen von Interesse, weil ihr am guten Ruf ihres Finanzplatzes sehr gelegen ist, und sie international nicht den Eindruck entstehen lassen will, sie könne als Drehscheibe für betrügerische Geschäfte missbraucht werden. Die nach Schweizerischem Strafgesetzbuch geltende Definition der Geldwäsche bleibt bestehen. AuBerdem schafft das Betrugsbekämpfungsabkommen auch keine neue Anmeldepflicht für die schweizerischen Finanzinstitute.

\section{Schlussfolgerungen}

Unser Beitrag analysierte die zweiten bilateralen Abkommen zwischen der Schweiz und der Europäischen Union im Gesamtzusammenhang ihrer Beziehungen. Unter diesem allgemeinen Blickwinkel offenbart sich ein Paradox: Obwohl diese neuen Verträge die Schweiz wiederum mehrere Schritte näher an die Europäische Union heranführen, rückt die Wahrscheinlichkeit eines Vollbeitritts der Eidgenossenschaft zur Union in weitere Ferne. ${ }^{23}$

Die Schweiz kann in Anbetracht ihrer geografischen Situation, des Risikos der wirtschaftlichen Diskriminierung und Abhängigkeit, aber auch der Gefahr der politischen Isolierung und Unsicherheit nicht abseits des politischen Systems der Europäischen Union stehen bleiben. Darum hat sie mit Hilfe der ersten bilateralen Abkommen und nun wiederum mit dem zweiten Paket derartiger Verträge versucht, das Abdriften in eine Randposition zu vermeiden. Die Mehrheit der Schweizer will momentan der Europäischen Union nicht beitreten. Trotzdem versucht die Schweiz, von den wirtschaftlichen und sicherheitspolitischen Vorteilen der europäischen Integration zu profitieren und gleichzeitig ihre Selbständigkeit soweit wie möglich zu bewahren. Solange dies außerhalb der Union möglich bleibt und von ihr akzeptiert wird, haben offensichtlich die meisten schweizerischen Bürger keine Bedenken, die Vorteile der Integration zu nutzen, ohne die allfälligen Nachteile mit zu tragen und den politischen Preis einer EU-Mitgliedschaft zu bezahlen. Deshalb verfolgt die Schweiz in Sachen europäische Integration seit mehr als 45 Jahren einen dritten Weg zwischen Beitritt und völligem Abseitsstehen. ${ }^{24}$

23 Dies gilt unter der Annahme, dass die Volksabstimmung vom 25.9.2005 betreffend die Ausdehnung des Personenfreizügigkeitsabkommens auf die neuen EU-Mitgliedstaaten angenommen wird. Sollte das Gegenteil eintreffen, ist momentan schwer abzuschätzen, wie sich die Beziehungen zwischen der Schweiz und der EU weiterentwickeln würden.

24 René Schwok: Ausblick 1992. Die Schweiz und der Europäische Wirtschaftsraum, Bern 1990. 
Nicht nur die Mehrheit der Bevölkerung sondern auch viele wirtschaftliche Interessenvertreter, der Schweizer Finanzplatz und ein Großteil der politischen Klasse stehen heute einem EU-Beitritt kritisch bis abweisend gegenüber. So hatten im März 2001 76,9 Prozent der Stimmbürger der Regierung ihre Zustimmung zur Aufnahme von Beitrittsverhandlungen mit der Europäischen Union verweigert, obwohl sie wussten, dass sie erneut über das Verhandlungsresultat und den EU-Beitritt hätten entscheiden können.

Angesichts der allgemeinen politischen Umstände und der dargestellten positiven Ergebnisse der zweiten bilateralen Abkommen kommen wir zum Schluss, dass der seit Anfang der 1990er Jahre verfolgte bilaterale Weg für die Schweiz maßgeschneiderte Resultate erbracht hat und es ihr erlaubt, die ihr am Herzen liegenden politischen und wirtschaftlichen Eigenarten beizubehalten. In der Tat kann sie damit am Schweizer Franken, am Bankgeheimnis, an der geringen indirekten Besteuerung sowie an ihrem Landwirtschaftsprotektionismus und ihren Kartellen in der Binnenwirtschaft festhalten.

Das Zinsbesteuerungsabkommen illustriert unsere Argumentation besonders trefflich. Es nähert die Schweiz der Europäischen Union in einem für beide Seiten sensiblen Bereich an. Aber gerade damit wird der EU-Beitritt für die Schweiz unattraktiver. Denn die grundsätzliche europäische Regelung in dieser Sache ist die Abschaffung des Bankgeheimnisses und die Einführung des automatischen Informationsaustausches betreffend die Besteuerung der Ersparnisse der in einem anderen Mitgliedstaat lebenden Bürger. Österreich, Belgien und Luxemburg haben jedoch eine Ausnahmestellung gegenüber dem Gemeinschaftsacquis erhalten. Aus strikt juristischer Sicht ist diese allerdings nur provisorischer und außerordentlicher Art, auch wenn sie faktisch unbefristet werden könnte.

Die gleichen Umstände und Überlegungen finden sich auch in Bezug auf den Euro. Großbritannien und Dänemark mussten der Wirtschafts- und Währungsunion nicht beitreten, weil sie zum Zeitpunkt ihrer Einführung bereits EU-Mitglieder waren und eine Opt out-Regelung erreichten. Ein neues Mitglied ist hingegen im Prinzip gehalten, den gesamten acquis communautaire zu übernehmen, den Euro sowie die Zins- und Mehrwertsteuerpolitik inbegriffen.

Nach dem In-Kraft-Treten der zweiten bilateralen Abkommen wird sich der Bundesrat in Anbetracht all dessen zweimal überlegen, ob er wirklich EU-Beitrittsverhandlungen in absehbarer Frist aufnehmen will. Der Schweizer Finanzplatz hat daran gewiss kein Interesse, denn die generelle Pflicht zur Übernahme des Gemeinschaftsacquis, welche definitionsgemäß solchen Verhandlungen zu Grunde liegt, würde zwangsweise Druckversuche zur Aufgabe des Bankgeheimnisses auslösen. Sowohl die Schweizer Regierung wie die Wirtschaftsverbände wissen nur zu gut, dass jede Verhandlung und jeder Deal ein gegenseitiges Geben und Nehmen mit sich bringen. Sie rechnen damit, dass im Bargainingprozess Druck ausgeübt wird, dass also ein direkter und meist auch ein indirekter Preis zu zahlen ist. Aus dieser Sicht lohnen die jetzt mit den ersten und zweiten bilateralen Abkommen vorgezeichneten maßgeschneiderten Verhältnisse bestimmt nicht ein neuerliches und vor allem ein allgemeines Öffnen der ,Büchse der Pandora', dem EU-Beitrittverhandlungen entsprächen.

Darum gilt das Paradox: Je länger sich die Schweiz der Europäischen Union mit bilateralen Abkommen annähert, umso weiter entfernt sie sich von einem Vollbeitritt! 